\title{
Protection Mechanism in Reliability Evaluation Approach to Multistate System with Common Cause Failure
}

\author{
Jinlei Qin $\mathbb{D}^{1,2}$ Zheng $\mathrm{Li} \mathbb{1}^{1},{ }^{1,2}$ and Youchan $\mathrm{Zhu}^{1,2}$ \\ ${ }^{1}$ Department of Computer, North China Electric Power University, Baoding, China \\ ${ }^{2}$ Engineering Research Center of Intelligent Computing for Complex Energy Systems, Ministry of Education, Baoding, China
}

Correspondence should be addressed to Zheng Li; yeziperfect@163.com

Received 2 December 2020; Revised 19 January 2021; Accepted 19 March 2021; Published 27 March 2021

Academic Editor: Abdel-Haleem Abdel-Aty

Copyright (c) 2021 Jinlei Qin et al. This is an open access article distributed under the Creative Commons Attribution License, which permits unrestricted use, distribution, and reproduction in any medium, provided the original work is properly cited.

Except the two types of state, complete failure and perfect functioning, some intermediate states also existed between those two states. This characteristic enables multistate system (MSS) whose components are often of more than two states. Moreover, because of dependence between components, common cause failure (CCF) enhances the failure risk of components during the operating period of MSS. A protection mechanism needs to be adopted because of the importance of certain components. For a MSS with CCF and protection mechanism, its reliability can be evaluated by a proposed approach. Certain components not only can cause failure at one state, but also can cause failure to other components because of the CCF. Under the impact of one type of $\mathrm{CCF}$, those components can constitute a specific group that can overlap with certain components. Universal generating function (UGF) is adopted to incorporate the CCF and protection mechanism of a component. Consequently, reliability indices of a MSS can be evaluated by the proposed approach based on UGF. Examples and comparisons illustrate and validate this method. This method can also help engineers prioritize elements that require protection mechanism under various resource limitations.

\section{Introduction}

With the development of industrial technology, product quality is being considered as an important aspect for customers. In addition, the traditional reliability theory cannot be applied in the modernized industrial system and/ or component because of its limitation to two states. For example, certain systems (such as those in the manufacturing, production, and oil and gas industry, and energy systems whose total performance can remain at different stages depending on the operating conditions) are referred to as multistate system (MSS) [1]. The analysis of MSS entails the development of new representation, modeling, and quantification technologies. A few researchers had focused on the above-mentioned aspects [2-5]. A new approach based on logical differential calculus is proposed for importance analysis and application of MSS, where MSS states change depending on simultaneous changing of some components' states $[6,7]$. Other technologies including these for assessment and optimization can be applied to MSSs in industries [8-11].
Multiple components fail owing to one cause, and this type of failure is named common cause failure (CCF). In real-life industry fields, it often occurred. Unprotected electronic equipment can experience outage in extreme weather conditions such as lightning events. And multiple failures of components often originate from voltage surge because of inappropriate switching in power system. CCFs can be divided into two types according to the origin of the cause: internal CCF (e.g., originating from propagated failures than from one component or multiple failures within system) and external CCF (e.g., abrupt variations in the environment such as those in the temperature and humidity or fire caused by lightning) [12, 13]. From the perspective of maintenance, an implicit two-stage approach was used to analyze the reliability of a nonrepairable MSS incorporating the CCF [14]. Using recursive and exact methods, the authors developed the reliability evaluation procedure for a nonrepairable system, wherein an individual component's failure could cause CCFs of other components [15]. However, in many real-life scenarios, repair is generally required to improve the reliability and/or availability of a 
system. An instantaneous availability model aimed at repairable MSSs with public bus performance sharing was suggested [16]. With regard to other aspects, such as the predictive inference of system reliability, a nonparametric approach was developed for a system with CCF [17].

CCF reduces the reliability of a technology system because of its interdependent failures (i.e., failures depend on other components). Interdependent failures are also called "interactive failure" and can be categorized into two types based on the consequences of failure of a component in the system: immediate failure dependence and gradual degradation dependence [18]. Life cycle cost and reliability were analyzed considering interdependent degradation $[19,20]$. The reliability analysis of MSS with statistics dependent components was studied from the perspective of statistics dependence that exists in the physical and complicated systems [21]. Owing to the inflection of element's performance distribution from those of other elements or subsystems, the dependency of elements is considered to evaluate MSS reliability based on universal generating function (UGF) technology [22]. Propagated failures are taken into account when a recursive approach was proposed for reliability analysis of MSS with CCF [15, 23]. System reliability can be improved using redundancies, fault detection, and isolation mechanisms. Nevertheless, once the above methods fail, certain components' failures cannot be detected. This phenomenon is named as imperfect fault cover (IFC). Stochastic multivalue models are put forward to achieve the availability of MSS with the incorporation of IFC [24]. An irrelevancy coverage model is also put forward to cover the irrelevant components, as well as the faulty components [25]. For assessing the risk of large cascading blackout, based on random chemistry, a new approach with high computational efficient is reported [26]. A dynamical stochastic is developed to explore the spread of flowing failure in the communication grid [27]. An MSS and component with CCF were modeled based on the dynamic Bayesian network to perform reliability assessment $[28,29]$. A reliability analysis method based on the evidence network was investigated to address the epistemic uncertainty of complex MSS with CCF [30]. Although many other researchers have focused on dependent failure during reliability assessment, inadequate consideration has been paid to the protection of important elements. Risk analysis was applied to reduce the failure risk of industrial systems in related fields [31-33]. Meanwhile, performance optimization and assessment of certain wireless networks and power line engineering were also investigated $[34,35]$. Although a rich body of research has been conducted for addressing effects of CCF in reliability analysis of diverse types of systems, protecting mechanism on certain important elements during the reliability assessment of an MSS with CCF is seldom focused on. One contribution is to build protecting mechanisms, which are implemented and extended for improvement of system reliability based on earlier studies. The other contribution is to develop a recursive procedure, which is based on the straightforward and conveniently programmable UGF technology. In this paper, the procedure aims to evaluate the reliability of an MSS with CCF under the protection measures implemented on certain important elements. Here, the protection measures can be considered as a special mechanism. For example, certain components need to be protected and isolated from fire caused by other components. A firewall would be set up to protect the important components.

The remainder of this paper is organized as follows. Section 2 presents the MSS model. The UGF method for estimating the system performance distribution and reliability indices is presented in Section 3. In addition, a specific method to evaluate an MSS with CCF under protection measures is developed in this section. Section 4 presents several examples to illustrate the validity of the approach. Section 5 concludes this paper.

\section{Modeling of MSS}

One of the most important characteristics of an MSS is the multiple states of its $n$ components, which are generally connected in series-parallel. The performance level $s$ of component $i$ can vary from zero (complete failure) to $m_{i}$ (perfect function). We denote each performance level by $h_{\mathrm{is}}$, and all the levels can form the vector $\mathbf{h}_{i}=\left\{h_{i 0}, h_{i 1}, \ldots\right.$, $\left.h_{i s}, \ldots, h_{i m_{i}}\right\}$. Furthermore, the performance levels of the overall system would adopt values from the following set, which is obtained as the Cartesian product of all $\mathbf{h}_{\mathbf{i}}$ :

$$
H^{n}=\mathbf{h}_{1} \times \mathbf{h}_{2} \times \cdots \times \mathbf{h}_{i} \times \cdots \times \mathbf{h}_{n} .
$$

The performance level of component $i$ at a specified moment will be also assumed to be a discrete random variable $H_{i} \in \mathbf{h}_{\mathbf{i}}$. In addition, $H_{i}$ would adopt the value $h_{\mathrm{is}}$ at a certain probability $p_{\text {is }}$ such that $p_{\text {is }}=\operatorname{Pr}\left\{H_{i}=h_{\text {is }}\right\}$. All the probabilities $p_{\text {is }}$ can constitute a set

$$
\mathbf{p}_{i}=\left\{p_{i 0}, p_{i 1}, \ldots, p_{i s}, \ldots, p_{i m_{i}}\right\} .
$$

Here, $\sum_{s=0}^{m_{i}} p_{\text {is }}=1$. That is, component $i$ could be in one, and only one, state at any time because all the $m_{i}+1$ states establish a group that is composed by mutually exclusive events.

Here, one assumption is that certain external reasons, such as lightning, which can cause failure or degradation of the system, will not be considered. Based on this situation, the state of an MSS can be ascertained explicitly from the state of its components.

The states of an MSS are denoted as $j \in\{0,1, \ldots, M\}$; i.e., the MSS would have $M+1$ states. Similarly, state 0 denotes complete failure, state $M$ denotes the perfect function, and the others denote the intermediate states. If $w_{j}$ denotes the performance level of an MSS at system state $j$, all the MSS performance levels can constitute the following set $L$ :

$$
L=\left\{w_{0}, w_{1}, \ldots, w_{j}, \ldots, w_{M}\right\} .
$$

Suppose that $W$ is a discrete random variable to denote certain performance level of MSS and can adopt values from $L$. Combining (1) and the above modeling of components and MSS, the system function of the MSS can be written as 


$$
W=\Phi\left(H_{1}, H_{2}, \ldots, H_{i}, \ldots, H_{n}\right): H^{n} \longrightarrow L .
$$

Essentially, this formula is to map the performance level space of components to the one of system. Likewise, the probability of system at certain performance level can be denoted by

$$
q_{j}=\operatorname{Pr}\left\{W=w_{j}\right\}, \quad j=0,1, \ldots, M .
$$

Several reliability indices can be derived from the above model to quantify the reliability of an MSS. In general, CCFs can be divided into two groups based on the origin of their causes (external and internal causes). CCFs belonging to the latter group are frequently referred to as propagated failures because these can propagate to other components of the same system. For the MSS considered here, component failure because of certain type of internal cause would result in the failure of other components. These failed components may be mutually dependent or even overlay on one or more components. Consequently, the performance level of the MSS would be weakened by an induced decrease in reliability. If the performance level reduces to a value below a boundary value $\xi$, which is generally named as the system demand, the reliability of the MSS cannot satisfy the demand and is considered unacceptable. In this scenario, the reliability of an MSS with CCF can be determined from the probability that the system satisfies $\xi$. From (5), the system reliability function can be determined as

$$
R(\xi)=\sum_{j=0}^{M} q_{j} \Delta\left(w_{j}, \xi\right)
$$

where $\Delta\left(w_{j}, \xi\right)=1, w_{j} \geq \xi ; \Delta\left(w_{j}, \xi\right)=0$, otherwise.

Another important index can be defined as the conditional expected performance $\Omega(\xi)$. This indicates the expected performance level of an MSS under the scenario where the MSS is in an acceptable state. This index can be obtained using the system reliability function $R(\xi)$ :

$$
\Omega(\xi)=\frac{\left(\sum_{j=0}^{M} q_{j} w_{j} \Delta\left(w_{j}, \xi\right)\right)}{R(\xi)} .
$$

To calculate the two indices, the distribution of the performance level of an MSS should be first obtained according to (5). The UGF technique has been demonstrated to be highly efficient for the MSS's reliability evaluation of different kinds of system structure. Particularly, for an MSS, whose components are connected by series or parallel, it is highly effective to implement the UGF technique by the recursive approach.

\section{UGF and Reliability Evaluation}

3.1. UGF Technique. The high-dimensional combinatorial problem is generally encountered during the process of calculating MSS reliability because of its multistate components. UGF technique can address this problem efficiently because UGF, also called a u-function or universal $z$ transform [36], is based on the generalization of the ordinary moment-generating function and $z$-transformation. For a multistate component $i$ at a state $g_{i}$ with a probability mass function (PMF) $p_{i g_{i}}$, its UGF can be defined by

$$
u_{i}(z)=\sum_{g_{i}=0}^{m_{i}} p_{i g_{i}} z^{h_{i g_{i}}}, \quad i=1,2, \ldots, n .
$$

The UGF of a series-parallel MSS can be determined by performing certain algebraic operations on the components' UGF. To represent the UGF of an MSS with the structure function (4), the composition operator $\otimes^{\phi}$ needs to be defined as follows by combining the UGF of each components:

$$
\begin{aligned}
U(z) & =\otimes^{\phi}\left(u_{1}(z), u_{2}(z), \ldots, u_{n}(z)\right) \\
& =\otimes^{\phi}\left(\sum_{g_{1}=0}^{m_{1}} p_{1 g_{1}} z^{h_{1 g_{1}}}, \sum_{g_{2}=0}^{m_{2}} p_{2 g_{2}} z^{h_{2 g_{2}}}, \ldots, \sum_{g_{n}=0}^{m_{n}} p_{n g_{n}} z^{h_{n g_{n}}}\right) \\
& =\sum_{g_{1}=0}^{m_{1}} \sum_{g_{2}=0}^{m_{2}} \ldots \sum_{g_{n}=0}^{m_{n}}\left(\prod_{i=0}^{n} p_{i g_{i}} z^{\phi\left(h_{1 g_{1}}, h_{2 g_{2}} \ldots, h_{n g_{n}}\right)}\right) .
\end{aligned}
$$

Here, the polynomial $U(z)$ represents the UGF of the MSS. The items on the right-hand side of the above formula are the feasible mutually exclusive combinations of the UGF of individual independent components. The function $\phi\left(h_{1 g_{1}}, h_{2 g_{2}}, \ldots, h_{n g_{n}}\right)$ needs to be determined according to the task characteristics.

The direct derivation of $U(z)$ is still challenging for different types of task. As presented in [36], exhibiting the $U(z)$ in a recursive method is effective from the perspectives of simplified calculation and derivation clarity. Particularly, for an MSS with complex configuration, the overall system can be represented as the composition of several subsystems consisting of certain components. This characteristic can be expressed as follows:

$$
\begin{aligned}
& \otimes^{\phi}\left(u_{1}(z), \ldots, u_{k}(z), u_{k+1}(z), \ldots, u_{n}(z)\right) \\
& =\otimes^{\phi}\left(\otimes^{\phi^{1}}\left(u_{1}(z), \ldots, u_{k}(z)\right), \otimes^{\phi^{2}}\left(u_{k+1}(z), \ldots, u_{n}(z)\right)\right) .
\end{aligned}
$$

Here, the functions $\phi, \phi^{1}$ and $\phi^{2}$ represent different operations for different configurations. The configuration of an MSS or its subsystems can generally be considered as the connection style among the subsystems or components. For any series-parallel components in subsystem, the composition operator can be employed recursively to obtain the UGF of the series-parallel components.

For continuous energy or material transmission system, such as electric power, oil, and water, its performance level can be defined as productivity. For paralleled components, the total productivity of the system is the sum of productivities of each individual component. The total productivity of two paralleled components ( $i$ and $j$ ) is equal to the sum of the individual productivities. The function $\phi^{p}$ calculates the sum of the corresponding performance. The performance of the two components in this occasion can be expressed as 


$$
\begin{aligned}
U_{i j}(z) & =u_{i}(z) \otimes \phi^{p} u_{j}(z) \\
& =\sum_{g_{i}=0}^{m_{i}} \sum_{g_{j}=0}^{m_{j}} p_{i g_{i}} p_{j g_{j}} z^{\operatorname{sum}\left(h_{i g_{i}}, h_{j g_{j}}\right)} .
\end{aligned}
$$

Similarly, for series components, the component that is of the minimum productivity would become the limit. Function $\phi^{s}$ calculates the minimum of all the parameters. Consequently, the UGF of two components ( $i$ and $j$ ) connected in series should adopt the following formula:

$$
\begin{aligned}
U_{i j}(z) & =u_{i}(z) \otimes \phi^{s} u_{j}(z) \\
& =\sum_{g_{i}=0}^{m_{i}} \sum_{g_{j}=0}^{m_{j}} p_{i g_{i}} p_{j g_{j}} z^{\min \left(h_{i g_{i}}, h_{j g_{j}}\right)} .
\end{aligned}
$$

\subsection{Expression of Component's UGF Including CCF. The CCF} triggered by component $i$ can correspond to state $0^{\prime}$ with the performance level $h_{i 0^{\prime}}=c$. Actually, the performance value can be equal to the component performance value of local failure with zero. If component $i$ is in state $0^{\prime}$, those components involved by this CCF would also be in the failure state with performance $c$. If a component cannot arouse CCF, the PMF of the corresponding state could be zeroed $p_{i 0^{\prime}}=0$. The UGF of component $i$ containing the CCF can be expressed by

$$
u_{i}(z)=\sum_{g_{i}=0}^{m_{i}} p_{i g_{i}} * z^{h_{i g_{i}}}+p_{i 0^{\prime}} * z^{c}
$$

The conditional PMF of component $i$ that cannot fail owing to the CCF can be denoted by

$$
u_{i}^{\prime}(z)=\sum_{g_{i}=0}^{m_{i}} \frac{p_{i g_{i}}}{1-p_{i 0^{\prime}}} * z^{h_{i g_{i}}}
$$

where $p_{i 0^{\prime}}$ is the probability of causing the CCF.

Let there be $b \leq n$ components that can cause the CCF simultaneously and independently. For the component vector $\beta=\{\beta(1), \beta(2), \ldots, \beta(r), \ldots, \beta(b)\}, r \in[1, b]$, the probability that the CCF originated from one component can be denoted by $p_{\beta(r) 0^{\prime}}$. Because these CCFs can occur independently, the result of combination is $2^{b}$.

For one combination $\delta,\left(0 \leq \delta \leq 2^{b}-1\right)$, the CCF comes from component $\beta(r)$ if

$$
\varepsilon(r)=\bmod _{2}\left\lfloor\frac{\delta}{2^{r-1}}\right\rfloor=1, \quad 1 \leq r \leq b .
$$

The group of components corresponding to the $\delta$ can be achieved after calculating the above formula from $\delta=0$ to $\delta=2^{b}-1$. The probability of the CCF corresponding to each $\delta$ is

$$
\phi_{\delta}=\prod_{r=1}^{b} p_{\beta(r) 0^{\prime}}^{\varepsilon(r)}\left(1-p_{\beta(r) 0^{\prime}}\right)^{1-\varepsilon(r)}, \quad\left(0 \leq \delta \leq 2^{b}-1\right) .
$$

The group of components causing CCF under the specific $\delta$ is

$$
\begin{aligned}
& v_{\delta}=\cup_{r=1}^{b} \gamma_{r}, \\
& \gamma_{r}= \begin{cases}\{\beta(r)\}, & \mathcal{E}(r)=1, \\
\varnothing, & \mathcal{E}(r)=0 .\end{cases}
\end{aligned}
$$

Assuming that a component fails owing to the CCF, the conditional PMF of its performance level can be denoted by $\psi(z)=z^{c}$. This shows that the component can only be in the failure state with performance level $c$.

When the CCF corresponding to the combination $\delta$ occurs, all the components in $v_{\delta}$ would be in CCF mode, and their UGFs would be substituted by $\psi(z)$. Conversely, the UGFs of components not belonging to $v_{\delta}$ must be denoted by (14).

3.3. Incorporating Protection into Component's UGF. Based on the properties of CCF, other failed components caused by component $\beta(r)$ can be expressed by $S_{\beta(r)}$. For each $\delta$, the group of components affected by the CCF can be obtained by

$$
\begin{aligned}
\mu_{\delta} & =\cup_{r=1}^{b} S_{\beta(r)}{ }^{\prime}, \\
S_{\beta(r)}^{\prime} & = \begin{cases}S_{\beta(r)}, & \varepsilon(r)=1, \\
\varnothing, & \varepsilon(r)=0 .\end{cases}
\end{aligned}
$$

For certain important components, protection measures can be adopted to safeguard the components from CCF. When the CCF corresponding to $\delta$ occurs, any component belonging to the group $\mu_{\delta}$ fails when its protection mechanism fails and continues functioning with its typical performance level when its protection mechanism functions well. The protection for component $i$ can fail with probability $\alpha_{i}$. Based on the whole probability theorem, protection can be incorporated in UGF as follows:

$$
\vec{u}_{i}(z)=\alpha_{i} * \psi(z)+\left(1-\alpha_{i}\right) * u_{i}^{\prime}(z) .
$$

Because of the protection, the UGF of a component in $\mu_{\delta}$ should be replaced with (19). Here, the performance level is generally equal to zero. That is, $\psi(z)=z^{0}$.

Then, the conditional PMF of system performance would be attained as $u_{\delta}(z)$. Combining (16), system UGF can be computed by

$$
U_{\text {sys }}(z)=\sum_{\delta=0}^{2^{b}-1} u_{\delta}(z) * \phi_{\delta}
$$

Using the expression of system's UGF, the relevant indices of reliability can be evaluated by (6) and (7).

In addition to the above analysis, the next steps should be adopted to realize the evaluation.

(i) S1. Integrate the CCF into component's UGF using (13).

(ii) S2. Achieve the conditional UGF $u_{i}^{\prime}(z)$ of component $i$ according to (14).

(iii) S3. Find out all the combinations of components evoked by the CCF. 
(iv) S4. For each combination $\delta,\left(0 \leq \delta \leq 2^{b}-1\right)$, fix the corresponding components, which can arouse the CCF based on (15).

(v) S5. Compute the probability of every combination $\delta$ by (16).

(vi) S6. Establish the set of components triggering CCF $v_{\delta}$ by (17), and substitute the UGF of all the components belonging to $v_{\delta}$ with $\psi(z)$.

(vii) S7. Determine the group of components influenced by the CCF given a combination $\delta$ according to (18). Furthermore, substitute the UGF of all the components belonging to the group $6 \mu_{\delta}$ with (19).

(viii) S8. Represent the conditional UGF $u_{\delta}(z)$ based on the physical structure of MSS.

(ix) S9. Obtain the UGF of system $U_{\text {sys }}(z)$ by (20).

After $U_{\text {sys }}(z)$ is achieved, the reliability indices can be evaluated using (6) and (7).

\section{Illustrative Examples}

4.1. Analytic Example. A type of series-parallel MSS shown in Figure 1 can be illustrated to validate the above method for the reliability evaluation of MSSs with CCF and protection. Flowing materials such as water, oil, or steam can be transported from A to B by the multistate components. The structure of system in this example is presented as follows. The MSS is configured by two subsystems: Sub1 and Sub2. Each subsystem is connected in series. Within each subsystem, these components are connected in parallel. Because of the CCF, the components $C_{12}$ and $C_{21}$ constitute the common group CG1. The component $C_{13}$ and the second subsystem form CG2. For example, a feeding water system consisting of pumps in a power plant can be divided into two subsystems. $C_{21}$ and $C_{22}$ are vital and need to be protected from the fire originating from $C_{12}$ and $C_{13}$.

Performance level of MSS in this example will depend on components' states and system configuration. The state number of each component and probabilities of these components at each state are listed in Table 1. For each component, the events of component in working, local failure, and failure to cause CCF constitute the total spaces. Thus, the sum of the probabilities in those states is one. However, the probability of protection failure is not dependent on the component and should be handled independently.

Based on the above analysis, the UGF of each component can be represented as

$$
\left\{\begin{array}{l}
u_{C_{11}}(z)=0.68^{*} z^{3}+0.25^{*} z^{5}+0.07^{*} z^{0} \\
u_{C_{12}}(z)=0.64^{*} z^{5}+0.25^{*} z^{8}+0.06^{*} z^{0}+0.05^{*} z^{c} \\
u_{C_{13}}(z)=0.65^{*} z^{3}+0.14^{*} z^{7}+0.15^{*} z^{0}+0.06^{*} z^{c} \\
u_{C_{21}}(z)=0.6^{*} z^{6}+0.2^{*} z^{10}+0.2^{*} z^{0} \\
u_{C_{22}}(z)=0.5^{*} z^{2}+0.35^{*} z^{6}+0.15^{*} z^{0}
\end{array}\right.
$$

Their conditional UGF can be obtained by (14) as follows:

$$
\left\{\begin{array}{l}
u_{C_{11}}^{\prime}(z)=u_{C_{11}}(z), \\
u_{C_{12}}^{\prime}(z)=0.6737^{*} z^{5}+0.2632^{*} z^{8}+0.0631^{*} z^{0}, \\
u_{C_{13}^{\prime}}^{\prime}(z)=0.6915^{*} z^{3}+0.1489^{*} z^{7}+0.1596^{*} z^{0}, \\
u_{C_{21}}^{\prime}(z)=u_{C_{21}}(z), \\
u_{C_{22}}^{\prime}(z)=u_{C_{22}}(z) .
\end{array}\right.
$$

The components causing CCF are $C_{12}$ and $C_{13}$, such that $\beta(1)=C_{12}, \beta(2)=C_{13}$. Therefore, the components affected by these are $S_{\beta(1)}=\left\{C_{21}\right\}, S_{\beta(2)}=\left\{C_{21}, C_{22}\right\}$. The parameters corresponding to different combinations are listed in Table 2 . These values of $\phi_{\delta}$ will be used to calculate the UGF of system according to $(20)$.

Because of the protection measures, according to (19), the UGF of $C_{21}$ and $C_{22}$ can be expressed as follows:

$$
\left\{\begin{array}{l}
\vec{u}_{C_{21}}(z)=0.3^{*} z^{0}+(1-0.3)^{*} u_{C_{21}}^{\prime}(z)=0.42^{*} z^{6}+0.14^{*} z^{10}+0.44^{*} z^{0} \\
\vec{u}_{C_{22}}(z)=0.4^{*} z^{0}+(1-0.4)^{*} u_{C_{22}}^{\prime}(z)=0.3^{*} z^{2}+0.21^{*} z^{6}+0.49^{*} z^{0}
\end{array}\right.
$$

According to the physical structure of the example, its conditional UGF can be obtained by

$$
u_{\delta}(z)=u_{\text {sub } 1}(z) \otimes^{\phi_{s}} u_{\text {sub2 }}(z)=\left(u_{C_{11}^{\prime}}^{\prime}(z) \otimes^{\phi_{p}} u_{C_{12}}^{\prime}(z) \otimes^{\phi_{p}} u_{C_{13}}^{\prime}(z)\right) \otimes^{\phi_{s}}\left(u_{C_{21}}^{\prime}(z) \otimes^{\phi_{p}} u_{C_{22}}^{\prime}(z)\right)
$$




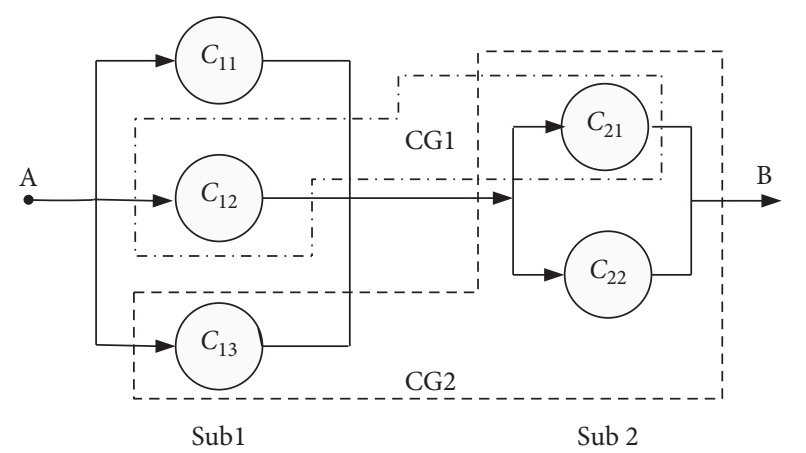

FIgURE 1: Series-parallel structure of MSS.

For $\delta=0$, there is no element in the sets $\nu_{\delta}$ and $\mu_{\delta}$. Therefore, in (24), no element's UGF needs to be substituted. There are 14 items in the UGF expression in the calculation result. The performance values and corresponding probabilities are displayed in Figure 2(a).

For $\delta=1, v_{\delta}=\left\{C_{12}\right\}$ and $\mu_{\delta}=\left\{C_{21}\right\}$. Therefore, $u_{C_{12}}^{\prime}(z)$ and $u_{C_{21}}{ }^{\prime}(z)$ in (24) should be substituted with $\psi(z)$ and $\vec{u}_{C_{21}}(z)$, respectively. Similarly, the 9 items in the result are displayed in Figure 2(b).

For $\delta=2, v_{\delta}=\left\{C_{13}\right\}$ and $\mu_{\delta}=\left\{C_{21}, C_{22}\right\}$. Therefore, $u_{C_{13}}{ }^{\prime}(z), u_{C_{21}}{ }^{\prime}(z)$ and $u_{C_{22}}{ }^{\prime}(z)$ in (24) should be substituted by $\psi(z), \vec{u}_{C_{21}}(z)$ and $\vec{u}_{C_{22}}(z)$, respectively. Similarly, there are 10 items in the UGF expression in the result as shown in Figure 2(c).

For $\delta=3, v_{\delta}=\left\{C_{12}, C_{13}\right\}$ and $\mu_{\delta}=\left\{C_{21}, C_{22}\right\}$. Therefore, in (24), $u_{C_{12}}{ }^{\prime}(z)$ and $u_{C_{13}}{ }^{\prime}(z)$ need to be substituted by $\psi(z)$. $u_{C_{21}}{ }^{\prime}(z)$ and $u_{C_{22}}{ }^{\prime}(z)$ should be substituted by $\vec{u}_{C_{21}}(z)$ and $\vec{u}_{C_{22}}(z)$, respectively. In the result, the UGF expression in the result has 4 items, as shown in Figure 2(d).

Finally, UGF of the whole system can be achieved by (20) like this:

$$
U_{s y s}(z)=\sum_{\delta=0}^{3} u_{\delta}(z) * \phi_{\delta} .
$$

The UGF expression of the whole system has 14 items and is shown in cyan color in Figure 3.

The reliability function of the entire system with protection is depicted in cyan color in Figure 4. From point A, we obtain $R(8)=0.6269$.

Similarly, the curve of the conditional expected performance of this system with protection is plotted in cyan color in Figure 5. Figure 5(a) is the entire graph of conditional expected performance, and Figure 5(b) shows details at $\xi=8$. From Point A, we obtain $\Omega(8)=9.7674$.

Although there are only five components in the system, the proposed approach is also suitable for system with a large number of components. This is because the entire procedure can be programmed conveniently using an ordinary programming language. For example, the total time required for the calculation using MATLAB in the computing environment applied by the authors is $1.9536 \mathrm{~s}$. The present main configuration of the computer is as follows: main frequency $=2.4 \mathrm{GHz}$ and memory $=4 \mathrm{~GB}$.
4.2. Comparison with the Cases without Protection. A comparison with the case without protection on the MSS can be performed to validate the effectiveness of the protection mechanism. The difference between the system with and without protection lies in the incorporation of the S6 and S7 in subsection $\mathrm{C}$ of Section 3. If an MSS has no protection mechanism, the elements in the set $\nu_{\delta} \cup \mu_{\delta}$ should be substituted by $\psi(z)$ while calculating $u_{\delta}(z)$. Based on the example in Section A, the procedure for calculating $u_{\delta}(z)$ is as follows:

For $\delta=0, \nu_{\delta} \cup \mu_{\delta}$ is empty. Therefore, the result does not vary with the result in the above example.

For $\delta=1, v_{\delta} \cup \mu_{\delta}=\left\{C_{12}, C_{21}\right\}$. Thus, the UGF of $C_{12}$ and $C_{21}, u_{C_{12}}^{\prime}(z)$ and $u_{C_{21}}{ }^{\prime}(z)$ in (24) should be substituted by $\psi(z)$. Its UGF expression in the result has 5 items.

For $\delta=2, v_{\delta} \cup \mu_{\delta}=\left\{C_{13}, C_{21}, C_{22}\right\}$. Therefore $u_{C_{13}}{ }^{\prime}(z)$, $u_{C_{21}}{ }^{\prime}(z)$, and $u_{C_{22}}{ }^{\prime}(z)$ in (24) should be substituted by $\psi(z)$. The result includes one item in the UGF as follows:

$$
u_{2}(z)=1 * z^{0} \text {. }
$$

For $\delta=3, v_{\delta}=\left\{C_{12}, C_{13}\right\}$ and $\mu_{\delta}=\left\{C_{21}, C_{22}\right\}$, and therefore $v_{\delta} \cup \mu_{\delta}=\left\{C_{12}, C_{13}, C_{21}, C_{22}\right\}$. In (24), $u_{C_{12}}^{\prime}(z)$, $u_{C_{13}}{ }^{\prime}(z), u_{C_{21}}^{\prime}(z)$ and $u_{C_{22}}^{\prime}(z)$ need to be substituted by $\psi(z)$. The result for this case is as follows:

$$
u_{3}(z)=1 * z^{0}
$$

Lastly, the UGF of whole system without protection will be obtained according to (20). There are also 14 items in its UGF, as shown in magenta in Figure 3.

In this case, its reliability function is plotted in magenta in Figure 4. Point B displays a lower value $(R(8)=0.6017)$ than that of Point A in Figure 4. Similarly, the curve of the conditional expected performance of the system at different performance levels is shown in magenta in Figure 5. A comparison of these curves reveals that these values are extremely close. We obtain $\Omega(8)=9.8110$ from Point B.

From the comparison results, it can be found that the reliability results of system with protection have higher values than the ones of the system without protection. This validates that the protection mechanism is effective and justified. Similarly, the curve of conditional expected performance of system without protection is higher than the one of the system with protection because it is inverse proportional to the reliability function. This also provides a proof to the effectiveness of protection.

4.3. Numerical Example. Here is another numerical example (with more number of components and CCFs) to prove the efficiency of the suggested approach. The MSS topology structure in this example is shown in Figure 6. This system consists of three subsystems: Sub1, Sub2, and Sub3. Sub1 and Sub2 are connected in series, and their combination is parallel to Sub3. There are three common groups: CG1, CG2, and CG3. The components in each common group are marked using dotted lines. In CG1, component $C_{11}$ causes $\mathrm{CCF}$, and $C_{21}$ is affected by the CCF. In addition, $C_{21}$ is also protected with a certain probability. In CG2, component $C_{12}$ 
TABLE 1: Parameters of components.

\begin{tabular}{|c|c|c|c|c|c|c|c|}
\hline \multirow{2}{*}{ Component } & \multicolumn{4}{|c|}{ Working state } & \multirow{2}{*}{$\begin{array}{c}\text { Local failure } \\
\text { Prob. }\end{array}$} & \multirow{2}{*}{$\begin{array}{l}\text { Failure that causes CCF } \\
\text { Prob. }\end{array}$} & \multirow{2}{*}{$\begin{array}{l}\text { Failure of protection } \\
\text { Prob. }\end{array}$} \\
\hline & Prob. & Tons/h & Prob. & Tons/h & & & \\
\hline$\overline{C_{11}}$ & 0.68 & 3 & 0.25 & 5 & 0.07 & - & - \\
\hline$C_{12}$ & 0.64 & 5 & 0.25 & 8 & 0.06 & 0.05 & - \\
\hline$C_{13}$ & 0.65 & 3 & 0.14 & 7 & 0.15 & 0.06 & - \\
\hline$C_{21}$ & 0.6 & 6 & 0.2 & 10 & 0.2 & - & 0.3 \\
\hline$C_{22}$ & 0.5 & 2 & 0.35 & 6 & 0.15 & - & 0.4 \\
\hline
\end{tabular}

TABle 2: Parameter values of different combinations $\delta$.

\begin{tabular}{|c|c|c|c|c|c|}
\hline \multirow{2}{*}{$\delta$} & \multicolumn{2}{|c|}{$\varepsilon(r)$} & \multirow[b]{2}{*}{$\phi_{\delta}$} & \multirow[b]{2}{*}{$v_{\delta}$} & \multirow[b]{2}{*}{$\mu_{\delta}$} \\
\hline & $r=1$ & $r=2$ & & & \\
\hline 0 & 0 & 0 & $=(1-0.05) \times(1-0.06)=0.893$ & $\varnothing$ & $\varnothing$ \\
\hline 1 & 1 & 0 & $=0.05 \times(1-0.06)=0.047$ & $\left\{C_{12}\right\}$ & $\left\{C_{21}\right\}$ \\
\hline 2 & 0 & 1 & $=(1-0.05) \times 0.06=0.057$ & $\left\{C_{13}\right\}$ & $\left\{C_{21}, C_{22}\right\}$ \\
\hline 3 & 1 & 1 & $=0.05 \times 0.06=0.003$ & $\left\{C_{12}, C_{13}\right\}$ & $\left\{C_{21}, C_{22}\right\}$ \\
\hline
\end{tabular}

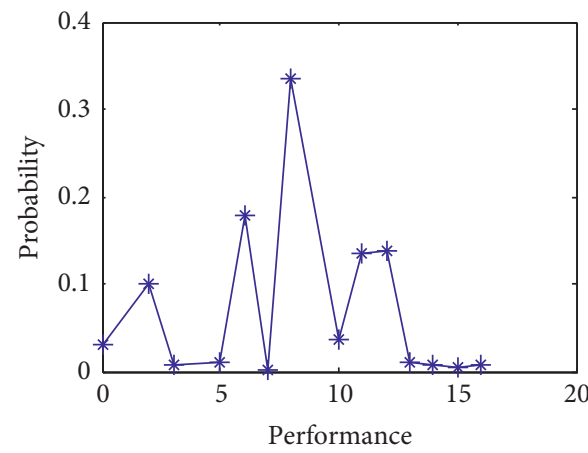

(a)

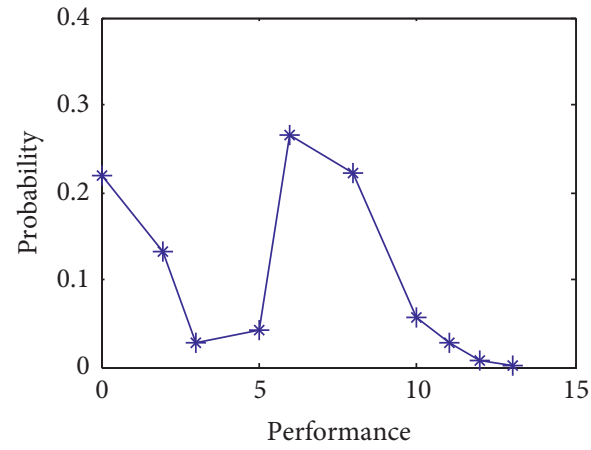

(c)

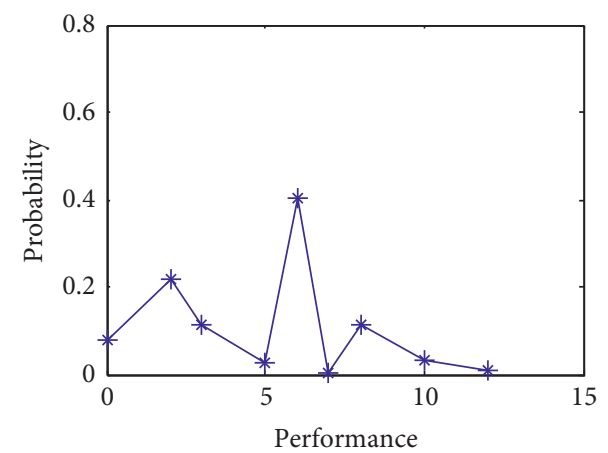

(b)

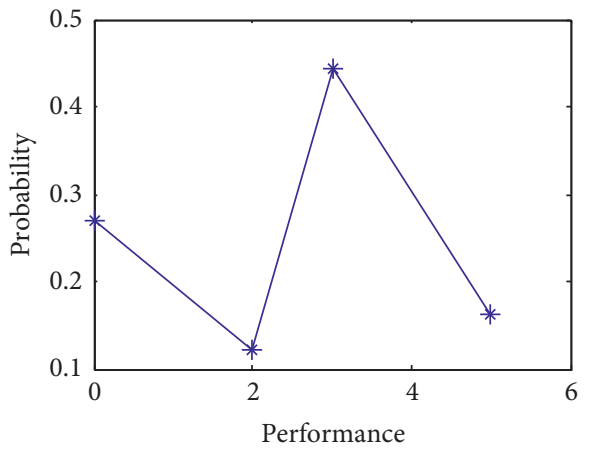

(d)

FIGURE 2: UGF results of four combinations.

triggers the CCF, and $C_{23}$ would fail and would also be protected. In CG3, component $C_{31}$ triggers the CCF and causes the failure of $C_{32}$ and $C_{33}$. In CG3, component $C_{33}$ would be protected with a certain probability.

Performance level of MSS will also vary with the states variation of components. All the states and probabilities of these components at each state in the system are recorded in Table 3.

The UGF of this system in different cases can be procured conveniently based on the proposed method. A comparison of these 26 items in the UGFs is depicted in Figure 7.
The reliability function of the system with and without protection can be compared. The results are displayed in Figure 8. Evidence shows that system with protection has a higher reliability level than that without protection. Point A has a higher value $(R(15)=0.6212)$ than that for Point $\mathrm{B}$ $(R(15)=0.5336)$. It also validates the effectiveness of protection against system safety.

In addition, there is no significant difference in conditional expected performance between the systems with and without protection. The whole graphs are shown in Figure 9(a). As shown in the figure, the values at Point A 


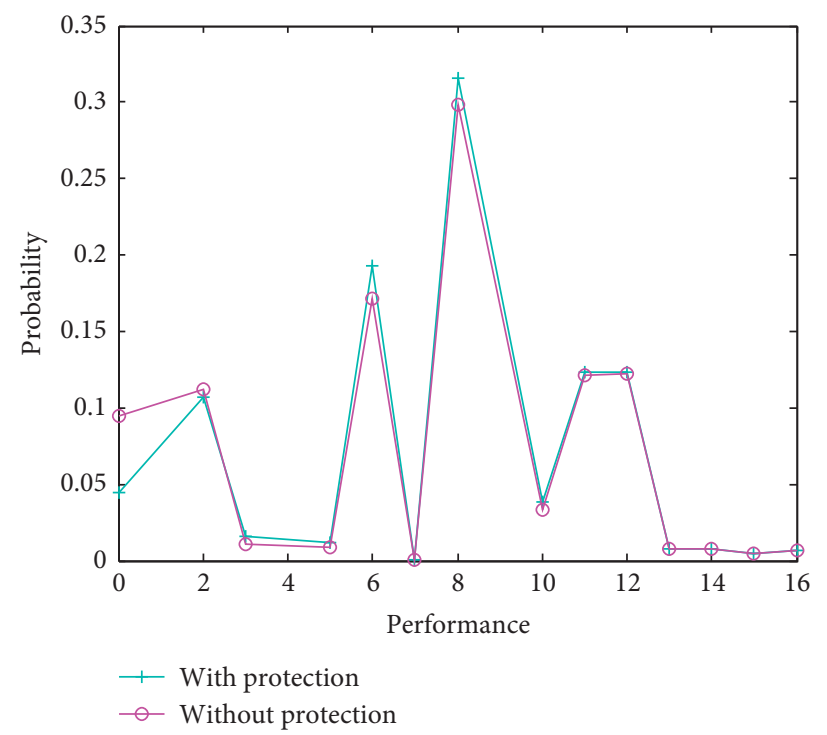

FIGURE 3: Comparison of UGF expression of the whole system with and without protection.

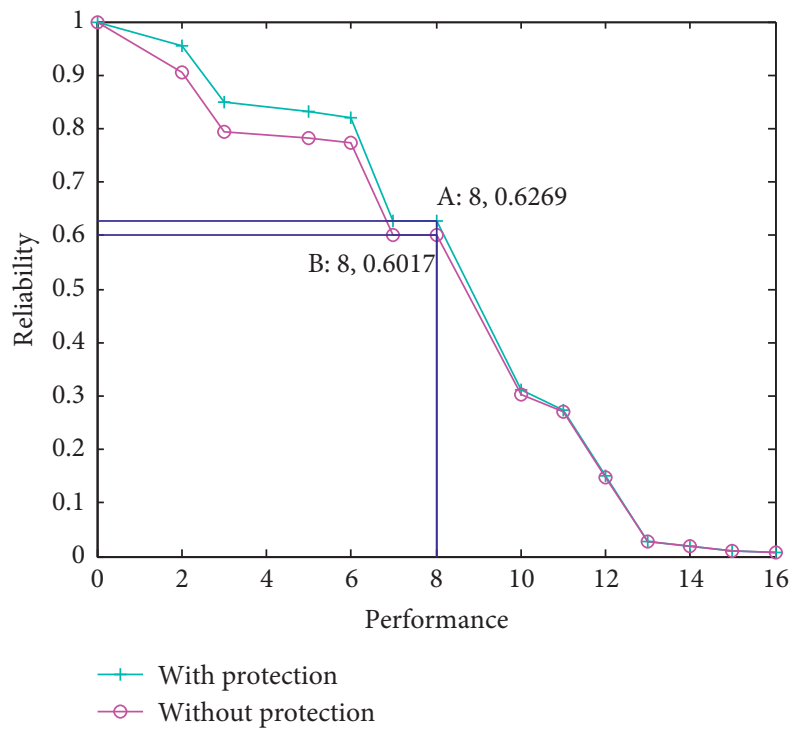

FIGURE 4: Reliability function of system with and without protection.

$(\Omega(15)=18.0709)$ and Point $\mathrm{B}(\Omega(15)=18.0633)$ are similar. Further details are shown in the local graphs in Figure 9(b).

The above analysis reveals that the recommended method is also suitable for a more complicated system with large-scale components. Furthermore, less time-consumption is another advantage over other similar methods. The time-consumption for this example under a configuration similar to that in Subsection A of Section 4 is $2.1484 \mathrm{~s}$. The time cost is generally reasonable for engineers. Based on the time-consumption results from the above two examples, this procedure implementing protection on important component is also acceptable in realistic engineering system.

In addition, the data or information of system coming from the computing result is crucial for reliability engineers. When making decision on which components need to be protected, those reliability indices provide scientific evidence. In particular, when there are many components in a large system, engineers can choose the most valuable components among the system and implement the protection on them based on the reliability evaluation indices. 


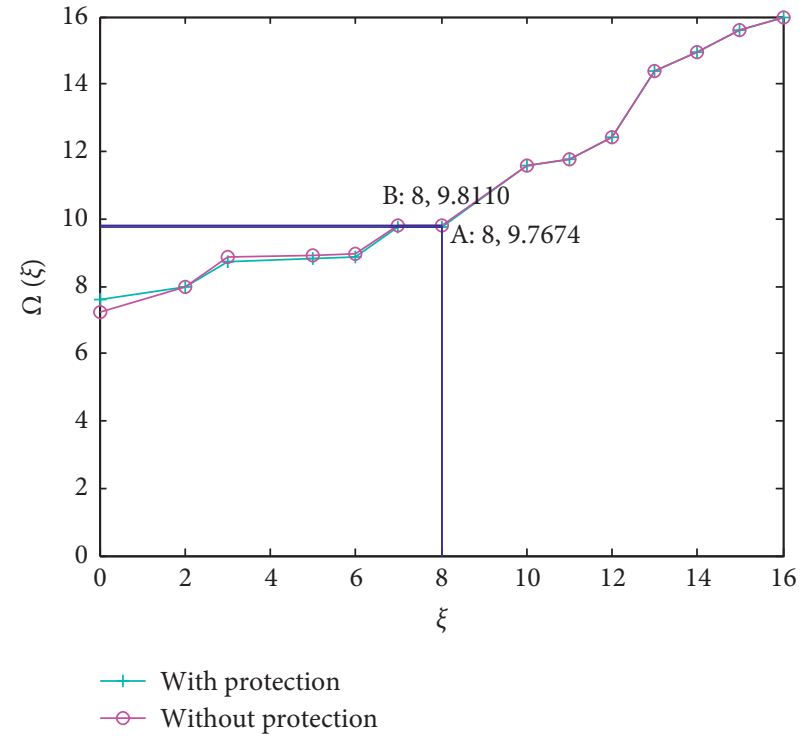

(a)

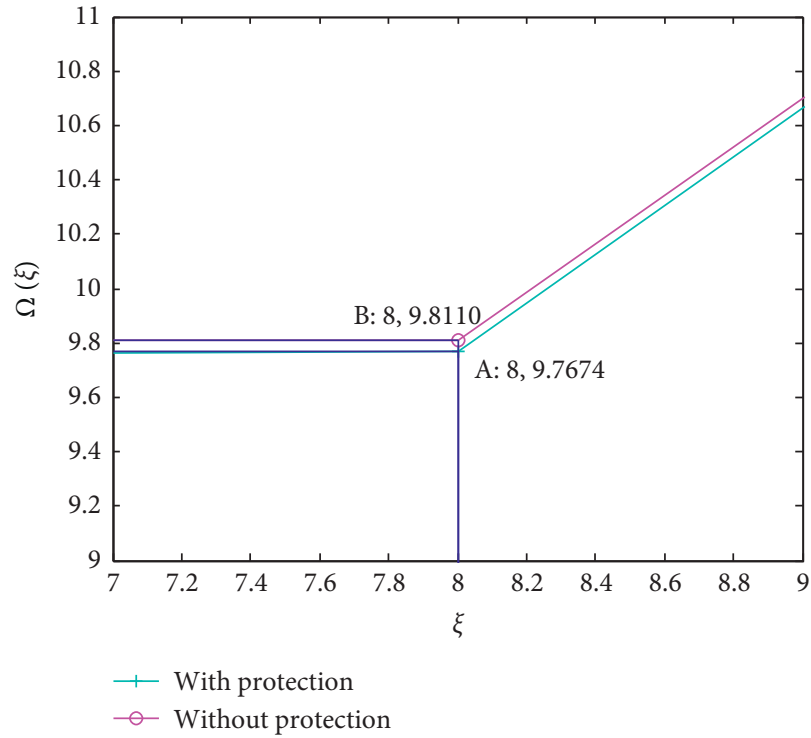

(b)

FIgURe 5: Conditional expected performance of system with and without protection. (a) The whole landscape. (b) The local landscape.

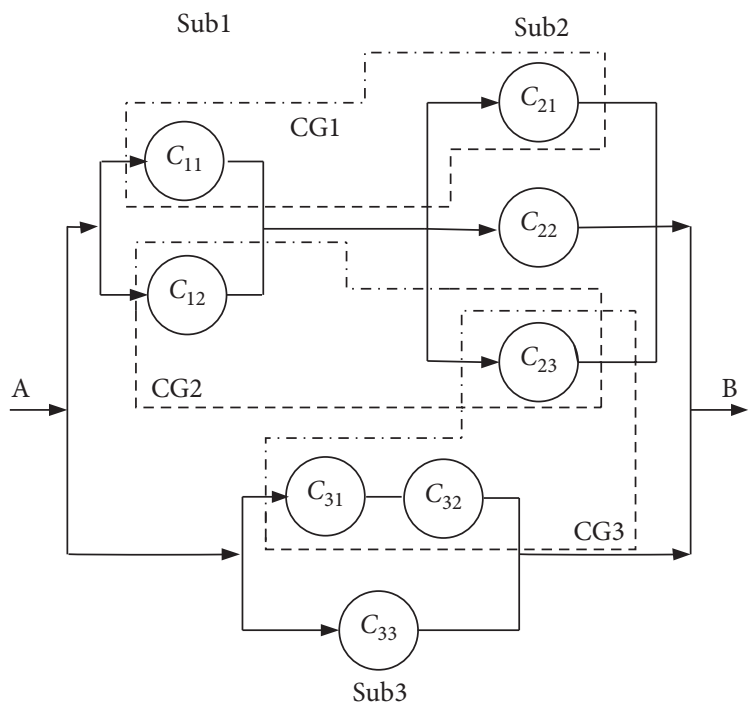

FIGURE 6: System topology of numerical example.

TABle 3: Parameters of all the components in the numerical example.

\begin{tabular}{|c|c|c|c|c|c|c|c|}
\hline \multirow{2}{*}{ Component } & \multicolumn{4}{|c|}{ Working state } & \multirow{2}{*}{$\begin{array}{c}\text { Local failure } \\
\text { Prob. }\end{array}$} & \multirow{2}{*}{$\begin{array}{c}\text { Failure to cause CCF } \\
\text { Prob. }\end{array}$} & \multirow{2}{*}{$\begin{array}{l}\text { Failure of protection } \\
\text { Prob. }\end{array}$} \\
\hline & Prob. & Tons/h & Prob. & Tons/h & & & \\
\hline$C_{11}$ & 0.62 & 4 & 0.25 & 5 & 0.07 & 0.06 & - \\
\hline$C_{12}$ & 0.64 & 5 & 0.25 & 9 & 0.06 & 0.05 & - \\
\hline$C_{21}$ & 0.71 & 5 & 0.14 & 7 & 0.15 & - & 0.2 \\
\hline$C_{22}$ & 0.6 & 7 & 0.2 & 10 & 0.2 & - & - \\
\hline$C_{23}$ & 0.63 & 4 & 0.22 & 6 & 0.15 & - & 0.3 \\
\hline$C_{31}$ & 0.65 & 4 & 0.14 & 7 & 0.15 & 0.06 & - \\
\hline$C_{32}$ & 0.7 & 7 & 0.2 & 10 & 0.1 & - & - \\
\hline$C_{33}$ & 0.55 & 3 & 0.3 & 6 & 0.15 & - & - \\
\hline
\end{tabular}




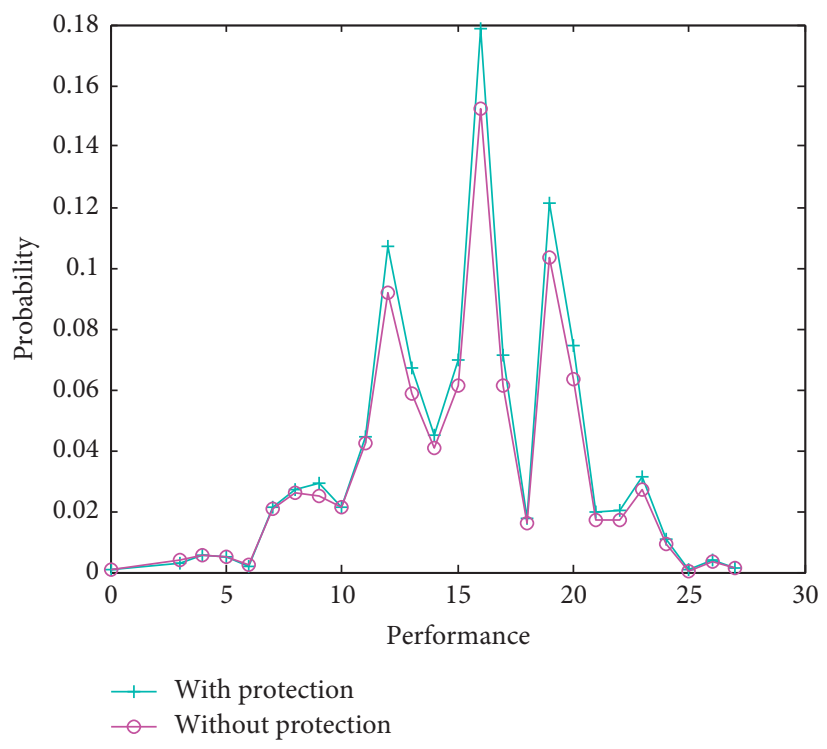

FIGURE 7: Comparison of items in the UGF under system with and without protection.

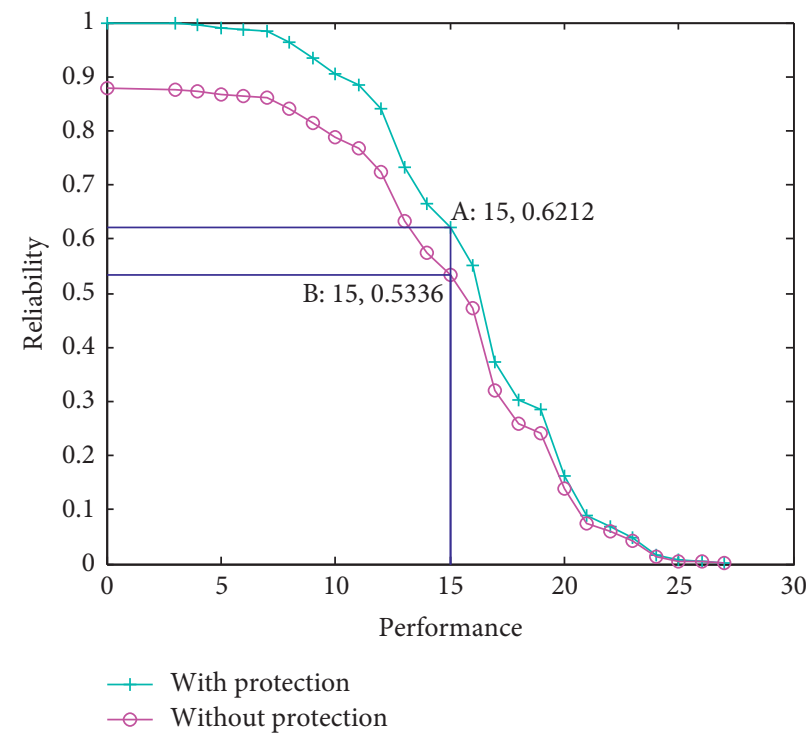

FIgURE 8: Comparison of reliability of system with and without protection. 


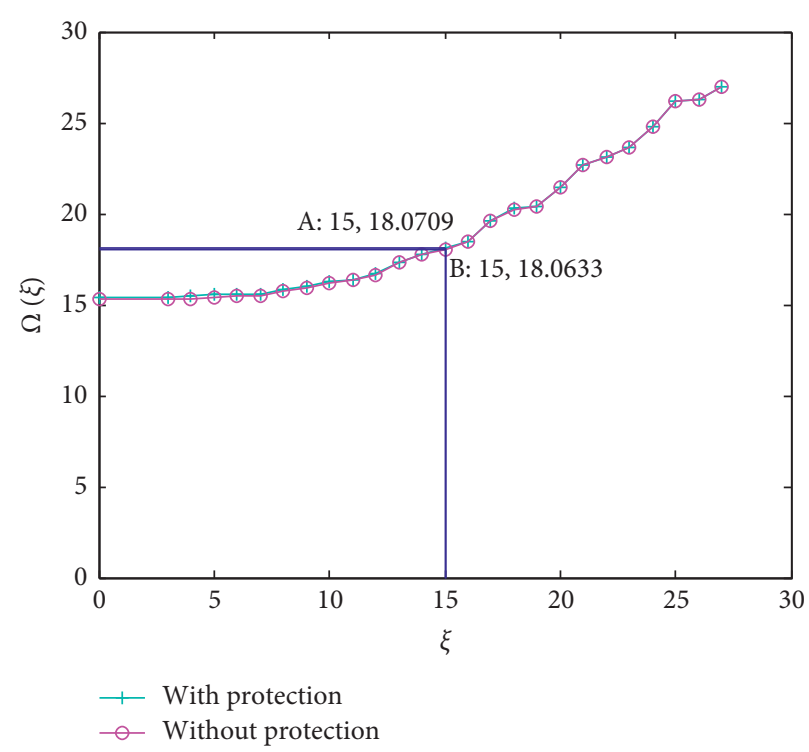

(a)

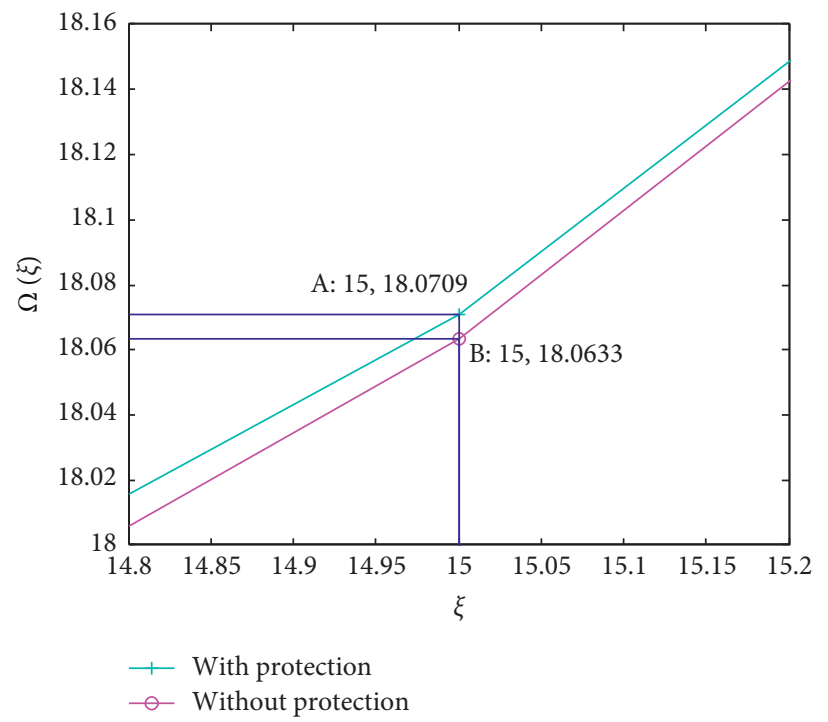

(b)

Figure 9: Conditional expected performance comparison. (a) The whole graphs. (b) The local graphs.

\section{Conclusion}

To evaluate the reliability of series-parallel MSSs with CCF and protection, a reliability evaluation approach is recommended in this paper. Components that can evoke the CCF may trigger failures of different subgroups of system components. These subgroups can also overlay with certain components. Because a few components are important for the system, the protection mechanisms are implemented on these ones. The factors of CCF and protection can be melted in the UGF based on the UGF technology. Because it is repeated for each combination, this approach can be programmed suitably by repetition. Some indices such as reliability and the expected conditional performance level can be calculated. To prove the validity of protection mechanism, a comparison with components without protection can be performed. This approach would also help in deciding which component is the most valuable and therefore, needs to be protected by reliability engineers. According to the assumptions in this approach, the restriction of system structure lies in the series-parallel MSS now. Probabilistic and fuzzy UGF would be considered to extend this approach in complex MSS such as bridge and $G(k / n)$ system in the future.

\section{Data Availability}

The data used to support the findings of this study are included within the article.

\section{Conflicts of Interest}

The authors declare that there are no conflicts of interest regarding the publication of this paper.

\section{Acknowledgments}

This work was supported financially in part by a grant from Fundamental Research Funds for the Central Universities (Nos. 2020MS120 and 2018MS076).

\section{References}

[1] E. Zio, "Reliability engineering: old problems and new challenges," Reliability Engineering \& System Safety, vol. 94, no. 2, pp. 125-141, 2009.

[2] J. Qin, Y. Niu, and Z. Li, “A combined method for reliability analysis OF multi-state system OF minor-repairable components," Ekspolatacja I Niezawodnosc - Maintenance and Reliability, vol. 18, no. 1, pp. 80-88, 2016.

[3] J. L. Qin, "Simulated method for reliability evaluation of multi-state coherent system," Iranian Journal of Science and Technology Transaction A-Science, vol. 42, no. A3, pp. 13631371, 2018.

[4] J. L. Qin and Z. Li, "Reliability and sensitivity analysis method for a multistate system with common cause failure," Complexity, vol. 2019, Article ID 6535726, 8 pages, 2019.

[5] J. Qin and Z. Li, "Reliability modeling for multistate system with preventive maintenance under customer demand," Complexity, vol. 2020, Article ID 3165230, 9 pages, 2020.

[6] M. Kvassay, E. Zaitseva, and V. Levashenko, "Importance analysis of multi-state systems based on tools of logical differential calculus," Reliability Engineering \& System Safety, vol. 165, pp. 302-316, 2017.

[7] P. Rusnak, "Logic differential calculus in time-dependent analysis of a pair of system components," in Proceedings of the 2018 IEEE 9th International Conference on Dependable Systems, Services and Technologies (DESSERT), Ukraine, Kyiv, May 2018.

[8] A. Lisnianski and G. Levitin, Multi-state System Reliability: Assessment, Optimization and Applications, World Scientific, Singapore, Singapore, 2003. 
[9] L. Anatoly, F. Ilia, and D. Yi, Multi-state System Reliability Analysis And Optimization For Engineers And Industrial Managers, Springer-Verlag, London, UK, 2010.

[10] Z. Li and J. Qin, "A modified particle swarm optimization with elite archive for typical multi-objective problems," Iranian Journal of Science and Technology, Transactions A: Science, vol. 43, no. 5, pp. 2351-2361, 2019.

[11] X. Huang, Y. Li, Y. Zhang, and X. Zhang, "A new direct second-order reliability analysis method," Applied Mathematical Modelling, vol. 55, pp. 68-80, 2018.

[12] L. Xing and G. Levitin, "BDD-based reliability evaluation of phased-mission systems with internal/external commoncause failures," Reliability Engineering \& System Safety, vol. 112, pp. 145-153, 2013.

[13] G. Levitin, L. Xing, S. Amari, and Y. Dai, "Reliability of nonrepairable phased-mission systems with common cause failures," Systems, Man, and Cybernetics: Systems, IEEE Transactions on, vol. 43, no. 4, pp. 967-978, 2013.

[14] G. Levitin, "Incorporating common-cause failures into nonrepairable multistate series-parallel system analysis," IEEE Transactions on Reliability, vol. 50, no. 4, pp. 380-388, 2001.

[15] G. Levitin, L. Xing, S. V. Amari, and Y. Dai, "Reliability of non-repairable phased-mission systems with propagated failures," Reliability Engineering \& System Safety, vol. 119, pp. 218-228, 2013.

[16] H. Yu, J. Yang, and H. Mo, "Reliability analysis of repairable multi-state system with common bus performance sharing," Reliability Engineering \& System Safety, vol. 132, pp. 90-96, 2014.

[17] F. P. A. Coolen and T. Coolen-Maturi, "Predictive inference for system reliability after common-cause component failures," Reliability Engineering \& System Safety, vol. 135, pp. 27-33, 2015.

[18] Y. Sun, L. Ma, J. Mathew, and S. Zhang, "An analytical model for interactive failures," Reliability Engineering \& System Safety, vol. 91, no. 5, pp. 495-504, 2006.

[19] B. Liu, P. Do, B. Iung, and M. Xie, "Stochastic filtering approach for condition-based maintenance considering sensor degradation," IEEE Transactions on Automation Science and Engineering, vol. 17, no. 1, pp. 177-190, 2020.

[20] B. Liu, "Life cycle cost analysis considering multiple dependent degradation processes and environmental influence," Reliability Engineering \& System Safety, vol. 197, p. 10, 2020.

[21] C. D. Dao and M. J. Zuo, "Reliability analysis of multi-state systems with s-dependent components," in Proceedings of the 2015 Annual Reliability and Maintainability Symposium (RAMS), Palm Harbor, FL, USA, January 2015.

[22] G. Levitin, "A universal generating function approach for the analysis of multi-state systems with dependent elements," Reliability Engineering \& System Safety, vol. 84, no. 3, pp. 285-292, 2004.

[23] G. Levitin, L. Xing, H. Ben-Haim, and Y. Dai, "Reliability of series-parallel systems with random failure propagation time," IEEE Transactions on Reliability, vol. 62, no. 3, pp. 637-647, 2013.

[24] X. Song, "A stochastic approach for evaluating the reliability of multi-stated phased-mission systems with imperfect fault coverage," in Proceedings of the 2017 Prognostics and System Health Management Conference (PHM-Harbin), Harbin, China, October 2017.

[25] J. Xiang, F. Machida, K. Tadano, and Y. Maeno, "An imperfect fault coverage model with coverage of irrelevant components," IEEE Transactions on Reliability, vol. 64, no. 1, pp. 320-332, 2015.
[26] P. Rezaei, P. D. H. Hines, and M. J. Eppstein, "Estimating cascading failure risk with random chemistry," IEEE Transactions on Power Systems, vol. 30, no. 5, pp. 2726-2735, 2015.

[27] W. Ren, J. Wu, X. Zhang, R. Lai, and L. Chen, "A stochastic model of cascading failure dynamics in communication networks," IEEE Transactions on Circuits and Systems II: Express Briefs, vol. 65, no. 5, pp. 632-636, 2018.

[28] B. Cai, Y. Liu, and Q. Fan, "Performance evaluation of subsea BOP control systems using dynamic Bayesian networks with imperfect repair and preventive maintenance," Engineering Applications of Artificial Intelligence, vol. 26, no. 10, pp. 2661-2672, 2013.

[29] J. H. Mi, "Reliability analysis OF multi-state system with common cause failure based ON bayesian networks," Eksploatacja I Niezawodnosc-Maintenance and Reliability, vol. 15, no. 2, pp. 169-175, 2013.

[30] J. Mi, Y.-F. Li, W. Peng, and H.-Z. Huang, "Reliability analysis of complex multi-state system with common cause failure based on evidential networks," Reliability Engineering \& System Safety, vol. 174, pp. 71-81, 2018.

[31] B. Fan, Y. Zeng, K. Jiang, and L. Tang, "A controllable chaotic immune algorithm for risk-aware routing in DiffServ networks," Computer Communications, vol. 76, pp. 67-76, 2016.

[32] C. B. Li, J. H. Yuan, and X. Feng, "Linguistic assessment information risky multi-criteria decision-making about wind power investment," Journal of Intelligent \& Fuzzy Systems, vol. 30, no. 5, pp. 3017-3023, 2016.

[33] C. Li, J. Yuan, D. Liu, W. Li, and S. Lin, "Risk group assessment based on the demands of important consumers with standby electric source," Journal of Intelligent \& Fuzzy Systems, vol. 33, no. 4, pp. 2177-2188, 2017.

[34] Y. Ju, J. Y. Yan, and H. Xu, "Performance optimization based on compressive sensing for wireless sensor networks," Wireless Personal Communications, vol. 95, no. 3, pp. 19271941, 2017.

[35] Z. X. Chen, "Performance analysis of dual-media cooperative communication based on wireless and power line under hybrid fading," International Journal of Distributed Sensor Networks, vol. 15, no. 5, p. 11, 2019.

[36] G. Levitin, The Universal Generating Function in Reliability Analysis and Optimization, Springer-Verlag, London, UK, 2005. 